University of Nebraska - Lincoln

DigitalCommons@University of Nebraska - Lincoln

Publications, Agencies and Staff of the U.S.

Department of Commerce

U.S. Department of Commerce

2010

Dynamics of Strongly Nonlinear Kinks and Solitons in a Two-Layer Fluid

K. Gorshkov

Russian Academy of Sciences

L. A. Ostrovsky

NOAA ESRL, lev.a.ostrovsky@noaa.gov

I. Soustova

Russian Academy of Sciences

Follow this and additional works at: https://digitalcommons.unl.edu/usdeptcommercepub

Part of the Environmental Sciences Commons

Gorshkov, K.; Ostrovsky, L. A.; and Soustova, I., "Dynamics of Strongly Nonlinear Kinks and Solitons in a Two-Layer Fluid" (2010). Publications, Agencies and Staff of the U.S. Department of Commerce. 272. https://digitalcommons.unl.edu/usdeptcommercepub/272

This Article is brought to you for free and open access by the U.S. Department of Commerce at DigitalCommons@University of Nebraska - Lincoln. It has been accepted for inclusion in Publications, Agencies and Staff of the U.S. Department of Commerce by an authorized administrator of DigitalCommons@University of Nebraska - Lincoln. 


\title{
Dynamics of Strongly Nonlinear Kinks and Solitons in a Two-Layer Fluid
}

\author{
By K. Gorshkov, L. A. Ostrovsky, and I. Soustova
}

\begin{abstract}
Perturbation theory is developed for interaction of strongly nonlinear solitary waves close to the limiting, tabletop solitons ( $\Pi$-solitons). The method is based on representing each soliton as a compound of two kinks so that the interaction of $N$ solitons is treated as the interaction of $2 N$ kinks. As an example the Miyata-Choi-Camassa equations for a two-layer fluid is considered. Equations for kink coordinates are obtained and analyzed. Some nontrivial features of two-soliton interaction characteristic of the strongly nonlinear case are established.
\end{abstract}

\section{Introduction}

Since 1960s an important, if not a central, role in the nonlinear wave theory was played by weakly nonlinear processes described by the model equations, such as Boussinesq, Korteweg-de Vries, Gardner, and Benjamin-Ono equations. However, in an increasing number of practical cases, the waves are strongly nonlinear so that the above equations are inapplicable. A typical example is that of internal waves in the ocean. It is a common knowledge now that in many cases the coastal internal waves are strongly nonlinear, i.e., characteristic amplitudes of vertical displacement are comparable with and even exceed the background values of vertical wave scale (e.g., the pycnocline depth). The relevant observational data are outlined in, e.g. [1-6] and in the review

Address for correspondence: L.A. Ostrovsky, NOAA ESRL, R/PSD99, 325 Broadway, Boulder, CO 80305; e-mail: lev.a.ostrovsky@noaa.gov 
paper [7]. Earlier models of such processes have used the Korteweg-de Vries $(\mathrm{KdV})$ equation modified by a cubic nonlinear term (the Gardner equation), which is still essentially weakly nonlinear, although it can work better than the "pure" $\mathrm{KdV}[2,5,8,9]$. For strongly nonlinear waves, besides the direct numerical simulation of the hydrodynamic equations, some attempts to construct strongly nonlinear, long-wave equations similar to the known weakly nonlinear models mentioned above, have been undertaken, mostly for the two-layer model of stratification [10-12]. In spite of more strong restrictions of applicability than that for weak nonlinearity, in many cases the long-wave, weakly dispersive models still provide a reasonably good approximation for strong waves, including solitons. A specifics of many such models is that with the increase of soliton amplitude, its profile, after narrowing at small amplitudes, then broadens up to a limiting tabletop shape corresponding to a pair of fronts (kinks) tending to indefinitely separate from each other when approaching the maximal (limiting) amplitude [10-14]. Numerical simulation of such processes as, e.g., interactions of two or more near-limiting solitons, even in the framework of simplified models, needs a high preciseness and thus can be time-consuming and even unstable. It is also important that numerical approach may not be able to provide a qualitative understanding of the general character of the process for different medium parameters and initial conditions.

Thus, it would be helpful to apply, when possible, an approximate approach that allows to further simplify the problem and even obtain analytical expressions for soliton parameters. Such an approach has been developed in 1980s for solitons in Ref. [15] and subsequent papers (see the review in [16]). It was shown that well separated solitons interact as classical particles with potentials corresponding to their asymptotics far from their centers. However, for near-limiting solitons this approach becomes, in general, ineffective because of their length so that their frontal and rear areas interact separately. In $[17,18]$ the perturbation theory was modified in such a way that a soliton is considered as a compound of two kinks interacting separately with another kinks. This approach was applied to an integrable Gardner equation that is applicable to weakly nonlinear waves but has a limiting soliton as a solution.

However, an advantage of such "direct" perturbation methods is that they are not limited by the integrability of an equation and thus can be applied to strongly nonlinear systems. Moreover, as only the wave asymptotics ("tails") are involved in the approximate equations, knowing an analytical expression for a soliton may not be necessary. In this paper the perturbation theory for kinks is applied to a system suggested by Miyata [10] and derived by Choi and Camassa [11] for strongly nonlinear, weakly dispersive waves in a two-layer fluid (in what follows we refer to it as the MCC equations). This system is a rather typical example of strongly nonlinear equations used for description of the oceanic internal waves. 


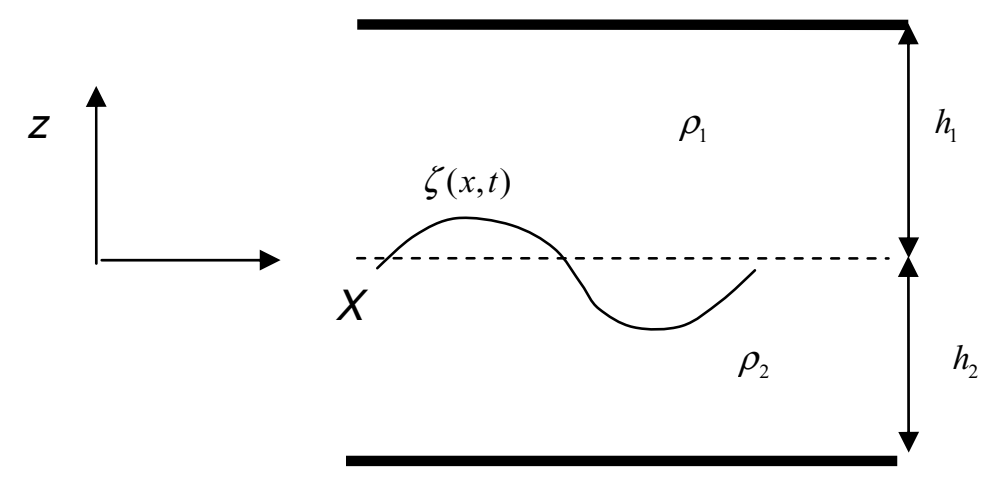

Figure 1. Internal wave in a two-layer liquid. This figure is colour online.

\section{Strongly nonlinear solitons and kinks}

We consider the MCC system for strongly nonlinear waves in a two-layer fluid in the form obtained in ref. [11]:

$$
\begin{aligned}
\eta_{1,2 t}+\left(\eta_{1,2} u_{1,2}\right)_{x} & =0 ; \\
\rho_{1,2}\left(u_{1,2 t}+u_{1,2} u_{1,2 x}+g \xi_{x}\right) & =-p_{x}+\frac{1}{3 \eta_{1,2}}\left(\eta_{1,2}^{3}\left(\frac{\partial}{\partial t}+u_{1,2} \frac{\partial}{\partial x}\right)^{2} \xi\right)_{x},
\end{aligned}
$$

where $\eta_{1,2}=h_{1,2} \mp \xi, h_{1,2}$ are undisturbed thicknesses of the upper and lower layers, respectively, $\xi(x, t)$ is vertical displacement of the interface between the layers; $u_{1,2}$ are horizontal fluid velocities (averaged over the vertical coordinate), $\rho_{1,2}$ are fluid densities, $p$ is pressure at the interface, and $g$ is gravity acceleration. The geometry of the problem is shown in Figure 1. In the system (1) the first pair of equations exactly follows from the continuity equations integrated over the vertical coordinate in each layer. The second pair describes variation of horizontal momentum in the long-wave approximation (the layers are thin as compared to the characteristic wavelength). Note that this system can also be derived from the Lagrangian approach [12].

Consider first stationary solutions of (1) which depend on a single variable $X=x-c t$, where $c=$ const. Then, after elementary transformations, the system (1) is reduced to a second-order ordinary differential equation (ODE) for the displacement $\xi$ :

$$
\begin{aligned}
& \qquad m(\xi) \xi_{x x}+\frac{1}{2} m_{\xi} \xi_{x}^{2}+W_{\xi}(\xi)=0 \\
& m(\xi)=\frac{\rho_{1} B_{1}^{2}}{3\left(h_{1}-\xi\right)}+\frac{\rho_{2} B_{2}^{2}}{3\left(h_{2}+\xi\right)} ; \quad w(\xi)=\frac{3}{2} m(\xi)-\frac{\rho_{2}-\rho_{1}}{2} g \xi^{2}+B_{3} \xi \\
& \text { where } m(\xi)=\frac{\rho_{1} B_{1}^{2}}{3\left(h_{1}-\xi\right)}+\frac{\rho_{2} B_{2}^{2}}{3\left(h_{2}+\xi\right)}, W(\xi)=\frac{3}{2} m(\xi)-\frac{\rho_{2}-\rho_{1}}{2} g \xi^{2}+B_{3} \xi \text { and } B_{1,2,3} \\
& \text { are integration constants. }
\end{aligned}
$$


We are interested in the family of solitary solutions of Equation (2) with the asymptotics $u(x \rightarrow \pm \infty)=0, \xi(x \rightarrow \pm \infty)=0$; this choice corresponds to the solitons discussed in, e.g., Ref. [13]. In this case $B_{1,2}=-c h_{1,2}$ and $B_{3}=\frac{3}{2} m(0)$. These solitons can be characterized by two independent parameters: the coordinate of the soliton center $S$ and its velocity $c$ or, equivalently, its amplitude $a$; the latter two parameters are related as

$$
c^{2}=c_{0}^{2}\left(h_{1}-a\right)\left(h_{2}+a\right) /\left(h_{1} h_{2}-c_{0}^{2} a / g\right) \text {, }
$$

where

$$
c_{0}^{2}=g h_{1} h_{2} \frac{\rho_{2}-\rho_{1}}{\rho_{1} h_{2}+\rho_{2} h_{1}}
$$

is square of the velocity of a long, linear internal wave. The possible values of the wave parameters lie within the intervals $c_{0}^{2}<c^{2}<c_{m}^{2}$ and $0<a^{2}<\xi_{m}^{2}$, where

$$
c_{m}^{2}=g\left(h_{1}+h_{2}\right) \frac{1-\left(\rho_{1} / \rho_{2}\right)^{1 / 2}}{1+\left(\rho_{1} / \rho_{2}\right)^{1 / 2}}, \quad \xi_{m}=\frac{h_{1}-h_{2}\left(\rho_{1} / \rho_{2}\right)^{1 / 2}}{1+\left(\rho_{1} / \rho_{2}\right)^{1 / 2}} .
$$

The polarity of a soliton depends on the layer thickness, namely, $a>$ 0 (pycnocline elevation) at $\left(h_{1} / h_{2}\right)>\sqrt{\rho_{1} / \rho_{2}}$ and $a<0$ (depression) at $\left(h_{1} / h_{2}\right)<\sqrt{\rho_{1} / \rho_{2}}$.

As already mentioned, there exists a limiting configuration; namely, at $c \rightarrow c_{m}$ the soliton length infinitely increases; at velocities close to $c_{m}$ it has a tabletop shape with an amplitude close to $\xi_{m}$ and relatively sharp edges - kinks. In what follows, we will sometimes refer to such configurations as $\Pi$-solitons. A single kink is a transition between two constant states. It has a unique velocity, $\xi=c_{m}$, and is characterized by a single parameter, $S$, which is the coordinate of its center. The corresponding solutions of (2) can be represented as

$$
\frac{V+1}{|V-1|}\left(\frac{\left|V-V_{m}\right|}{V+V_{m}}\right)^{V_{m}}=A e^{ \pm \Lambda_{0}(X-S)} .
$$

Here $X=x-c_{m} t, S$ is the coordinate of the kink, which is defined by the relation $\xi(X=S)=\frac{\xi_{m}}{2}$. Other variables and parameters in (6) are

$$
\begin{gathered}
V=\sqrt{\left(1+\xi / a_{*}\right)}, \quad V_{m}=\sqrt{\left(1+\xi_{m} / a_{*}\right)}, \quad a_{*}=\frac{h_{1} h_{2}\left(\rho_{1} h_{1}+\rho_{2} h_{2}\right)}{\rho_{1} h_{1}^{2}-\rho_{2} h_{2}^{2}}, \\
\Lambda_{0}^{2}=\frac{3 g\left(\rho_{2}-\rho_{1}\right) \xi_{m}^{2}}{c_{m}^{2}\left(\rho_{2} h_{2}^{2}-\rho_{1} h_{1}^{2}\right)}, \quad A=\left(\frac{V_{1 / 2}+1}{\left|V_{1 / 2}-1\right|}\right)\left(\frac{\left|V_{1 / 2}-V_{m}\right|}{V_{1 / 2}+V_{m}}\right)^{V_{m}}, \\
V_{1 / 2}=\sqrt{\left(1+\xi_{m} / 2 a_{*}\right)} .
\end{gathered}
$$

The signs + and - in (6) refer to the kinks of different polarities corresponding to transitions from $\xi=0, u_{1,2}=0$ at $(X-S) \rightarrow+\infty$ to $\xi=\xi_{m}, u_{1,2}=U_{1,2}=\mp c_{m} \xi_{m}\left(h_{1,2} \mp \xi_{m}\right)$ (frontal kink, $\left.\xi_{f}\right)$ at $(X-S) \rightarrow-\infty$ 


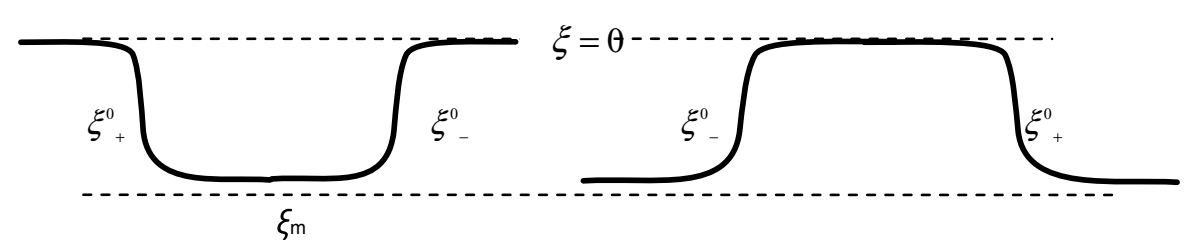

Figure 2. Stationary $\Pi$-solitons in Equation (2) containing kinks.

and back from $\xi=\xi_{m}, u_{1,2}=U_{1,2}$ at $(X-S) \rightarrow+\infty$ to $\xi=0, u_{1,2}=0$ (rear kink, $\left.\xi_{r}\right)$. Note that $1+\xi / a_{*}>0$ at all admissible values of $\xi$, within the limits $-h_{2}<\xi<h_{1}$.

According to (6), the behavior of kinks near their asymptotics $\xi=0$ and $\xi=\xi_{m}$ is exponential:

$$
\xi_{f, r}=\left\{\begin{array}{l}
e_{0} e^{\mp \Lambda_{0}(X-S)} \text { at }(X-S) \rightarrow \pm \infty \\
\xi_{m}+e_{m} e^{ \pm \Lambda_{m}(X-S)} \text { at }(X-S) \rightarrow \mp \infty
\end{array}\right.
$$

Here the upper and lower signs correspond to the kinks $\xi_{f}$ and $\xi_{r}$, respectively, and

$$
\begin{aligned}
& e_{0}=\mp\left|a_{*}\right| V_{m} A^{-1}\left(\frac{\left|V_{m}-1\right|}{V_{m}+1}\right)^{V_{m}}, \quad e_{m}= \pm\left|a_{*}\right| V_{m}\left(A \frac{\left|V_{m}-1\right|}{V_{m}+1}\right)^{1 / V_{m}}, \\
& \Lambda_{m}=\Lambda_{0} / V_{m} .
\end{aligned}
$$

In these expressions the upper and lower signs correspond to $\xi_{m}<0$ and $\xi_{m}>0$, respectively. Qualitatively, the above solutions are analogous to those of the Gardner equation, the interaction of which is considered in [18]. In particular, a П-soliton can be represented as a compound of two kinks of opposite polarities (Figure 2):

$$
\xi_{s}(x, t) \approx \xi_{f}\left(x-c_{m} t-S_{f}\right)+\xi_{r}\left(x-c_{m} t-S_{r}\right)-\xi_{m} ; S_{f}>S_{r} .
$$

This solution will be used as a basic one to be perturbed to find the coordinates of kinks and perturbation of their velocities. Note that transposing these kinks so that

$$
\xi_{s}(x, t) \approx \xi_{f}\left(x-c_{m} t-S_{f}\right)+\xi_{r}\left(x-c_{m} t-S_{r}\right)-\xi_{m} ; S_{f}<S_{r},
$$

we obtain a solution of opposite polarity but with nonzero asymptotics $\xi \rightarrow \xi_{m}, u_{1,2} \rightarrow U_{1,2}$.

An important difference between the kinks considered here and those in the Gardner model is that the latter have the same exponential asymptotics at both ends, whereas in the former these asymptotics have different exponents at $X-S \rightarrow \pm \infty$, i.e., $\Lambda_{0} \neq \Lambda_{m}$. As will be shown below, this circumstance results in a specific behavior of phase shifts upon interaction. 


\section{Interaction of solitons close to limiting}

As previously mentioned, adequate description of solitons close to limiting can be achieved by treating soliton interaction as the interaction of kinks of alternate polarities. For definiteness, it is assumed that solitons (and kinks) propagate along $x$ in positive direction $\left(c_{m}>0\right)$, so that soliton fronts are formed by the kinks with even numbers, and their trailing edges by the odd-numbered kinks.

To apply the perturbation theory, we suppose that the ensemble of kinks is "rarefied" so that the distances between the centers of kinks, $S_{i}-S_{i-1}$, are large in comparison with the characteristic width of the kinks, which is of the order of $\Lambda_{0, m}^{-1}$. This means that the kinks interact weakly and the resulting variations of their velocities are much smaller than their unperturbed velocities $\xi_{m}$. The small parameter of the problem, $\varepsilon$, has an order of the ratio $\left|\max \dot{S}_{i}\right| / c_{m}$. An algorithm for finding an approximate solution for an ensemble of interacting solitons begins with representing a solution in the vicinity of each, $i$ th kink as an expansion

$$
\begin{gathered}
u_{i(1,2)}(x, t)=u_{i(1,2)}^{(0)}\left(X-S_{i}\right)+\sum_{n=1}^{\infty} u_{i(1,2)}^{(n)}\left(X-S_{i}, \tau, \rho\right), \\
\xi_{i}(x, t)=\xi_{i}^{(0)}\left(X-S_{i}\right)+\sum_{n=1}^{\infty} \xi_{i}^{(n)}\left(X-S_{i}, \tau, \rho\right),
\end{gathered}
$$

followed by matching these solutions in areas between the kinks. In these expansions, $X=x-c_{m} t ; \xi_{i}^{(0)}$ and $u_{i(1,2)}^{0}$ are stationary (with respect to $X$ ) solutions of (2) corresponding to the kinks with the unknown, slowly varying coordinates $S_{i}(\rho, \tau)$, where $\rho=\varepsilon x, \tau=\varepsilon t$ are "slow" variables. The variables $u_{i(1,2)}^{(n)}$ and $\xi_{i}^{(n)}$ are the $n$th order perturbations. Substitution of (12) into the basic system (1) and using expansion in powers of $\varepsilon$, result in the standard perturbation scheme. In each order, the perturbations $u_{i(1,2)}^{(n)}$ and $\xi_{i}^{(n)}$ at $n \geq 1$ can be found as functions of $X-S_{i}$ from the ODE linearized with respect to an $i$ th kink, whereas matching of these solutions provides equations for the coordinates $S_{i}$ and, in general, for integration constants.

This problem can be solved in quadratures:

$$
\begin{aligned}
u_{i(1,2)}^{(n)}= & b_{i(1,2)} \xi_{i}^{(n)}+H_{i(1,2)}^{(n)}+d_{i(1,2)} U_{i(1,2)}^{(n)} \\
\xi_{i}^{(n)}\left(X-S_{i}\right)= & f_{i 1}\left(C_{i}^{(n)}-\int^{X-S_{i}} f_{i 2}\left(H_{i}^{(n)}+G_{i}^{(n)}\right) d X\right) \\
& +f_{i 2}\left(D_{i}^{(n)} \int^{X-S_{i}} f_{i 1}\left(H_{i}^{(n)}+G_{(i)}^{(n)}\right) d X\right) .
\end{aligned}
$$


Here $U_{i 1,2}^{(n)}(\tau, \rho), G_{(i)}^{(n)}, C_{i}^{(n)}$, and $D_{i}^{(n)}$ are integration constants in a $n$th approximation, whereas the functions $H_{i(1,2)}^{(n)}$ depend on the perturbations $u_{i(1,2)}^{(n)}$ and $\xi_{i}^{(n)}$ and on integration constants found in previous approximations; $b_{i(1,2)}=\frac{c_{m} h_{1,2}}{\left(\eta_{i(1,2)}^{(0)}\right)^{2}}, \quad d_{i(1,2)}=\frac{h_{(1,2)}}{\eta_{i(1,2)}^{(0)}}, \quad \eta_{i 1,2}^{(0)}=h_{1,2} \mp \xi_{i}^{(0)}\left(X_{i}-S_{i}\right)$. The functions $f_{i 1}=\xi_{i X}^{(0)}$ and $f_{i, 2}=\xi_{i X}^{(0)} \int^{X} m^{-1}\left(\xi_{i}^{(0)}\right)\left(\xi_{i X}^{(0)}\right)^{-2} d X$ are linearly independent solutions of the homogeneous equation $\hat{L}_{i} f_{i}=0$, where $\hat{L}_{i}=\frac{d}{d X} m\left(\xi_{i}^{(0)}\right) \frac{d}{d X}+$ $\bar{W}\left(\xi_{i}^{(0)}\right)$. This is a linearized Equation (2) with $\bar{W}\left(\xi_{i}^{(0)}\right)=W_{\xi \xi}\left(\xi_{i}^{(0)}\right)+m_{\xi}^{\prime}\left(\xi_{i}^{(0)}\right)$ $\xi_{i X X}^{(0)}+\frac{1}{2} m_{\xi}^{\prime \prime}\left(\xi_{i}^{(0)}\right)\left(\xi_{i X}^{(0)}\right)$.

The partial solutions $f_{i 1}$ are localized functions of $X-S_{i}$ with exponentially decaying asymptotics (see (7)), whereas the functions $f_{i 2}\left(X-s_{i}\right)$ have the asymptotics exponentially increasing in both directions

$$
f_{i 2}\left(X-S_{i}\right)=- \begin{cases}{\left[2 e_{0} \Lambda_{0}^{2} m(0)\right]^{-1} e^{ \pm \Lambda_{0}\left(X-S_{i}\right)},} & X-S_{i} \rightarrow \pm \infty \\ {\left[2 e_{m} \Lambda_{m}^{2} m\left(\zeta_{m}\right)\right]^{-1} e^{\mp \Lambda_{m}\left(X-S_{i}\right)},} & X-S_{i} \rightarrow \mp \infty\end{cases}
$$

Here the upper and lower signs \pm refer to the even and odd numbers $i$, respectively.

\section{The main-order approximation}

We begin with matching the kinks in the zero approximation. Their unperturbed asymptotics $\xi_{\mathrm{m}}$ are matched automatically between the kinks of alternating polarities. Thus, the lower-order global solution $\xi_{N}^{(0)}(x, t)$ has the form of a superposition

$$
\xi_{N}^{(0)}(x, t)=\sum_{i=1}^{2 N} \xi_{i}^{(0)}\left(X-S_{i}\right)-N \xi_{m}
$$

Note that the exponential asymptotics (7) are of the order of $\exp \left(-\Lambda_{0, m}\left|S_{i \pm 1}-S_{i}\right|\right)$ near the neighboring kink centers, and they should be matched only in the next approximation. It should be stressed that asymptotics of neighboring kinks exponentially increase with the distance from the center of the given $i$ th kink. There are no such terms in (7) but they appear in the first approximation. Indeed, in the first order the right-hand parts of Equation (13) are 


$$
\begin{aligned}
H_{i(1,2)}^{(1)} & =\mp \frac{\zeta_{i}^{(0)}}{\eta_{i(1,2)}^{(0)}} \frac{d S_{i}}{d t}, \quad H_{i}^{(1)}+G_{(i)}^{(1)}=H_{i I}^{(1)}+H_{i I}^{(1)}, \\
H_{i I}^{(1)} & =\frac{2}{c_{m}} g\left(\rho_{2}-\rho_{1}\right) \zeta_{i}^{(0)} \frac{d S_{i}}{d t}-\frac{c_{m} \rho_{1} h_{1}}{\left(\eta_{i 1}^{(0)}\right)^{2}} U_{i 1}^{(1)}+\frac{c_{m} \rho_{2} h_{2}}{\left(\eta_{i 2}^{(0)}\right)^{2}} U_{i 2}^{(1)}+G_{i}^{(1)}, \\
H_{i I I}^{(1)} & =\frac{c_{m}}{\xi_{i X}^{(0)}} \frac{d}{d X}\left[-c_{m} m\left(\xi_{i}^{(0)}\right) \frac{\partial S_{i}}{\partial x}-\frac{\rho_{1} h_{1}}{\eta_{i 1}^{(0)}} U_{i 1}^{(1)}-\frac{\rho_{2} h_{2}}{\eta_{i 2}^{(0)}} U_{i 2}^{(1)}\right]\left(\xi_{i X}^{(0)}\right)^{2} .
\end{aligned}
$$

Here $\frac{d}{d t}=\frac{\partial}{\partial t}+c_{m} \frac{\partial}{\partial x}$.

As follows from (16), function $H_{i}^{(1)}\left(X-S_{i}\right)$ is bounded, and at $X-S_{i} \rightarrow \pm \infty$ it tends to nonzero constants (which may depend on "slow" variables). This function causes the asymptotics of the perturbation $\xi_{i}^{(1)}$ which, in general, contain both exponentially increasing and decreasing terms, as well as terms $\bar{\xi}_{i}^{(1)}$ tending to constants. Both the terms $\xi_{i}^{(1)}$ and $\bar{\xi}_{i}^{(1)}$ must be matched, whereas exponentially decreasing terms are small values of a higher order and should be matched in the next approximation. Note that the part of the solution $\xi_{i}^{(1)}$ induced by the term $H_{i I I}^{(1)}$ is of the latter type.

The exponentially increasing terms in $\xi_{i}^{(1)}$ exist due to the terms factored by $f_{i 2}$ in (13). Indeed, according to the asymptotics (16) and (17), exponential growth of the integral $\int f_{i 2} H_{i I}^{(1)} d X$ is neutralized by the decrease of the corresponding functions $f_{i 1}$, so that the first term in (15) remains limited: at $X-S_{i} \rightarrow \pm \infty$ it tends to constants. However, as $f_{i 2}$ increases and the integral $\int f_{i 1} H_{i I}^{(1)} d X$ tends to nonzero constants at $X-S_{i} \rightarrow \pm \infty$, it results in exponential divergence of both the free $\left(\sim D_{i}^{(1)}\right)$ and the forced part of the solution related to the right-hand side of (14). This diverging part of $\xi_{i}^{(1)}$ must be matched with the main exponential asymptotics of neighboring kinks $\xi_{i+1}^{(0)}$, which also have increasing asymptotics with respect to the center of the (ith) kink. As the exponent powers $\left(\Lambda_{0}\right)$ and $\left(\Lambda_{m}\right)$ of the main terms in expansions of the functions $\xi_{i+1}^{(0)}$ and $f_{i 2}$ coincide in the corresponding areas, a matching can be made by direct equalizing of the asymptotic values:

$$
\begin{aligned}
\left.\zeta_{i}^{(1)}\left(X-S_{i} \rightarrow+\infty\right)\right|_{\exp } & =\left.f_{i 2}\left(X-S_{i} \rightarrow+\infty\right)\right|_{\exp } \cdot\left(D_{i}^{(1)}+\int_{0}^{+\infty} f_{i 1} H_{i}^{(1)} d X\right) \\
& =\left.\zeta_{i+1}^{(0)}\left(X-S_{i+1} \rightarrow-\infty\right)\right|_{\exp } \\
\left.\zeta_{i}^{(1)}\left(x-s_{i} \rightarrow-\infty\right)\right|_{\exp } & =\left.f_{i 2}\left(x-s_{i} \rightarrow-\infty\right)\right|_{\exp } \cdot\left(D_{i}^{(1)}+\int_{0}^{-\infty} f_{i 1} H_{i}^{(1)} d X\right) \\
& =\left.\zeta_{i-1}^{(0)}\left(X-S_{i-1} \rightarrow+\infty\right)\right|_{\exp },
\end{aligned}
$$


where subscript «exp» denotes the main exponential term in the asymptotic series.

The part of perturbation $\xi_{i}^{(1)}$ which at $X-S_{i} \rightarrow \pm \infty$ tends to some constants $\bar{\xi}_{i}^{(1)}( \pm \infty)$, is matched with similar asymptotics of perturbations $\xi_{i \pm 1}^{(1)}$ in the neighboring kinks. The simplest way to find limits $\bar{\xi}_{i}^{(1)}\left(X-S_{i} \rightarrow \pm \infty\right)=\bar{\xi}_{i \pm}^{(1)}$ is to directly use the equation $\hat{L}_{i} \xi_{i}^{(n)}=H_{i}^{(n)}+G_{i}^{(n)}$, which has the solution (13). It is easy to conclude that $\bar{\xi}_{i}^{(1)}$ satisfies the equation

$$
\tilde{W}\left(\xi_{i}^{(0)}\left(X-S_{i} \rightarrow \pm \infty\right)\right) \cdot \bar{\xi}_{i}^{(1)}( \pm \infty)=H_{i I}^{(1)}( \pm \infty)
$$

Since $\tilde{W}\left(\xi_{i}^{(0)}\left(X-S_{i} \rightarrow+\infty\right)\right)=\tilde{W}\left(\xi_{i+1}^{(0)}\left(X-S_{i+1} \rightarrow-\infty\right)\right)$, matching of $\bar{\xi}_{i}^{(1)}$ и $\bar{\xi}_{i+1}^{(1)}$ is achieved simply by equating the right-hand parts of (16) with each other:

$$
H_{i I}^{(1)}\left(X-S_{i} \rightarrow+\infty\right)=H_{i+1 . I}^{(1)}\left(X-S_{i+1} \rightarrow-\infty\right) .
$$

In addition to the functions $\xi_{i}^{(1)}$, matching must be made for the perturbations of velocities $u_{i(1,2)}^{(1)}$ in each layer of the fluid. From comparison of expressions (13) connecting $u_{i(1,2)}^{(1)}$ with $\xi_{i}^{(1)}$ and $u_{i \pm 1(1,2)}^{(1)}$ with $\xi_{i \pm 1}^{(1)}$ при $X-S_{i \pm 1} \rightarrow \mp \infty$ it follows that:

(a) If the conditions (17) are met, increasing asymptotics of $u_{i(1,2)}^{(1)}$ at $X-S_{i} \rightarrow \pm \infty$ are identically matched with the main asymptotics of kink solutions for velocities $u_{i \pm 1(1,2)}^{(0)}$.

(b) The constant asymptotic limits of perturbations $u_{i(1,2)}^{(1)}$ are matched with the corresponding asymptotics of perturbations $u_{i \pm 1(1,2)}^{(1)}$ if the following conditions are met:

$$
\left.\left[H_{i(1,2)}^{(1)}+\frac{U_{i(1,2)}^{(1)} h_{1,2}}{\eta_{i(1,2)}^{(0)}}\right]\right|_{X-S_{i} \rightarrow+\infty}=\left.\left[H_{i+1(1,2)}^{(1)}+\frac{U_{i+1(1,2)}^{(1)} h_{1,2}}{\eta_{i+1(1,2)}^{(0)}}\right]\right|_{X-S_{i+1} \rightarrow-\infty} .
$$

After substituting Equation (16) for $H_{i(1,2)}^{(1)}$ and $H_{i I}^{(1)}$ into the matching Equations (17), (19), and (20), and performing elementary integration, we obtain equations for the functions $S_{i}, U_{i(1,2)}^{(1)}$, and $G_{i}^{(1)}$, which are of the main physical interest here:

$$
\frac{d S_{i}}{d t}=I_{i}+2\left\{\begin{array}{ll}
M_{0} e^{-\Lambda_{0}\left(S_{i}-S_{i-1}\right)}-M_{m} e^{-\Lambda_{m}\left(S_{+1 i}-S_{i}\right)}, & \text { odd } i \\
M_{m} e^{-\Lambda_{m}\left(S_{i}-S_{i-1}\right)}-M_{0} e^{-\Lambda_{0}\left(S_{i+1}-S_{i}\right)}, & \text { even } i
\end{array}\right\}
$$




$$
\begin{gathered}
G_{i+1}^{(1)}=G_{i}^{(1)}-\left\{\begin{array}{ll}
\xi_{m} q \frac{d\left(S_{i+1}-S_{i}\right)}{d t}, & \text { odd } i \\
0, & \text { even } i
\end{array}\right\}, \\
U_{i+1(1,2)}^{(1)}=U_{i(1,2)}^{(1)} \pm\left\{\begin{array}{ll}
\xi_{m} q \frac{d\left(S_{i+1}-S_{i}\right)}{d t}, & \text { odd } i \\
0, & \text { even } i
\end{array}\right\}\left(+ \text { refers to } U_{i(1)}^{(1)},- \text { to } U_{i(2)}^{(1)}\right), \\
M_{m}=\frac{m\left(\xi_{m}\right) e_{m}^{2} \Lambda_{m}^{3 / 2}}{g\left(\rho_{2}-\rho_{1}\right) \xi_{m}^{2} \Lambda_{0}^{1 / 2},} q=\frac{2 g\left(\rho_{2}-\rho_{1}\right)}{c_{m}}-\frac{c_{m} \rho_{1} h_{1}}{\left(\eta_{1}^{(0)} \xi(m)\right)^{2}}-\frac{c_{m} \rho_{2} h_{2}}{\left(\eta_{2}^{(0)}\left(\xi_{m}\right)\right)^{2}}, \\
M_{0}=\frac{m(0) e_{0}^{2} \Lambda_{0}^{3 / 2}}{g\left(\rho_{2}-\rho_{1}\right) \xi_{m}^{2} \Lambda_{m}^{1 / 2},} \quad \\
I_{i}=-\frac{\left(\Lambda_{0} \Lambda_{m}\right)^{1 / 2}}{g\left(\rho_{2}-\rho_{1}\right) \xi_{m}} G_{i}^{(1)}+\frac{c_{m} \rho_{1}\left(\Lambda_{0} \Lambda_{m}\right)^{-1 / 2}}{g\left(\rho_{2}-\rho_{1}\right) \xi_{m} \eta_{1}^{(0)} \xi(m)} U_{i(1)}^{(1)} \\
\frac{\rho_{2}\left(\Lambda_{0} \Lambda_{m}\right)^{-1 / 2}}{g\left(\rho_{2}-\rho_{1}\right) \xi_{m} \eta_{2}^{(0)} \xi(m)} U_{i(2)}^{(1)}
\end{gathered}
$$

Here and below we use the same notations, $t$ and $x$, for the dimensionless time and coordinate:

$$
t \rightarrow c_{m} \sqrt{\Lambda_{o} \Lambda_{m}} t, \quad x \rightarrow \sqrt{\Lambda_{0} \Lambda_{m}} x .
$$

This set of equations can be simplified in the following way. Substituting the last expression for $I_{i}$, into the first Equation (21), we then express the functions $G_{i}^{(1)}$ and $U_{i(1,2)}^{(1)}$ via the same functions of the previous number $i-1$. Repeating this procedure inductively, we finally obtain

$$
\frac{d S_{i}}{d t}=I_{1}(\tau, \rho)-2\left\{\begin{array}{ll}
M_{0} e^{-\Lambda_{0}\left(S_{i}-S_{i-1}\right)}+M_{m} e^{-\Lambda_{m}\left(S_{i+1}-S_{i}\right)}, & i \text {-odd } \\
M_{m} e^{-\Lambda_{m}\left(S_{i}-S_{i-1}\right)}+M_{0} e^{-\Lambda_{0}\left(S_{i+1}-S_{i}\right)}, & i-\text { even }
\end{array}\right\} .
$$

\section{The general solution and its features}

Equations (21) and (22) allow several important conclusions. First, using (22) to write the differences $S_{2 p}-S_{2 p-1}$ which are the distances between kinks forming the same soliton, and summing, we obtain

$$
\frac{d}{d t} \sum_{p=1}^{N}\left(S_{i=2 p}-S_{i=2 p-1}\right)=0, \quad p=1,2, \ldots, N,
$$


i.e., the sum of lengths of all solitons is an integral of motion. Then, summation in the expressions (21) for $G$ and $U$ yields

$$
\begin{gathered}
G_{2 N}^{(1)}(\tau, \rho)=G_{1}^{(1)}(\tau, \rho)-\xi_{m} q \frac{d}{d t} \sum_{p=1}^{N}\left(S_{2 p}-S_{2 p-1}\right)=G_{1}^{(1)}(\tau, \rho) \\
U_{2 N(1,2)}^{(1)}=U_{1(1,2)}^{(1)} \pm \xi_{m} \frac{d}{d t} \sum_{p=1}^{N}\left(S_{2 p}-S_{2 p-1}\right)=U_{1(1,2)}^{(1)} .
\end{gathered}
$$

Using the latter relations in the expressions (18) for the asymptotics $\bar{\xi}_{i}^{(1)}( \pm \infty)$, we obtain

$$
\begin{aligned}
\bar{\xi}_{1}^{(1)}(-\infty, \tau, \rho) & =\tilde{W}^{-1}\left(\zeta_{1}^{(0)}=0\right) H_{1}^{(1)}\left(\xi_{1}^{(0)}=0, G_{1}^{(1)}, U_{1(1,2)}^{(1)}\right) \\
& =\tilde{W}^{-1}\left(\zeta_{2 N}^{(0)}=0\right) H_{2 N I}^{(1)}\left(\xi_{2 N}^{(0)}=0, G_{2 N}^{(1)}, U_{2 N(1,2)}^{(1)}\right) \\
& =\bar{\xi}_{2 N}^{(1)}(+\infty, \tau, \rho) .
\end{aligned}
$$

Similar relations can be established for $\bar{u}_{1,(1,2)}^{(1)}(-\infty)$ and $\bar{u}_{2 N,(1,2)}^{(1)}(+\infty)$. The fields $\bar{\xi}_{2 N}^{(1)}(+\infty)$ and $\bar{u}_{2 N,(1,2)}^{(1)}(+\infty)$ defined in the region in front of the soliton ensemble $\left(X>S_{2 N}\right)$, are prescribed in this problem (in particular, they may be zeros). Equation (24) means that no additional perturbations of the given fields $\bar{\xi}_{2 N}^{(1)}(+\infty)$ and $\bar{u}_{2 N,(1,2)}^{(1)}(+\infty)$ arise behind the soliton ensemble due to interactions. Thus, in the first approximation there is no radiation from the collision region, although it may appear in higher orders as the MCC system is probably not fully integrable.

General solution of the PDEs $(22)$ for $S_{i}(x, t)$ is obtained in the next section. Note that at $I=0$, this solution is closely connected with the general solution of the ODE system following from (22) after replacing the operator $\partial / \partial t+c_{m} \partial / \partial x$ by the ordinary derivative $d / d t$. The solution of the ODE system, $S_{i}\left(t,\left\{T_{j}\right\}\right)$, contains $2 N$ arbitrary constants denoted here as $\left\{T_{j}\right\}, j=1,2, \ldots 2 N$. The solution of the PDE system (22) has the same form, with changing of $T_{i}$ to $T_{j}(X)$. Functions $T_{j}(X)$ can be defined at $t \rightarrow-\infty$ where each pair of functions $x-S_{i}, i=2 p, 2 p-1$, describes phase variables $\Lambda_{p}\left(x-c_{p} t \pm \Delta_{p}\right)$ corresponding to a nonperturbed soliton propagating with a velocity $c_{p}$. For a $\Pi$-soliton close to limiting, the dependence $\Delta_{p}\left(c_{p}\right)$ is more important than $\Lambda_{p}\left(c_{p}\right)$. For that reason, in what follows we neglect the dependence of $S_{i}$ on $x$ and use the solution of the ODE for $S_{i}\left(t,\left\{T_{j}=\right.\right.$ const $\left.\}\right)$. Specifically, we will discuss the "pure" $N$-soliton solutions at $\bar{\xi}_{2 N}^{(1)}(+\infty), \bar{u}_{2 N,(1,2)}^{(1)}(+\infty)=0$, when $I_{1}=0$ in (22).

It is worth noting that the transformation

$$
R_{i}=\left\{\begin{array}{ll}
\Lambda_{m}\left(S_{i+1}-S_{i}\right)-\ln \left(\Lambda_{0} M_{m}\right), & i=2 p-1 \\
\Lambda_{0}\left(S_{i+1}-S_{i}\right)-\ln \left(\Lambda_{m} M_{0}\right), & i=2 p
\end{array} \quad p=1,2, \ldots N\right.
$$


reduces the system (22) to the known integrable equations of the "Langmuir chain" [19]:

$$
\frac{d R_{i}}{d t}=2\left(e^{-R_{i-1}}-e^{-R_{i+1}}\right)
$$

These equations have a universal form, independent of specific values of the parameters $M_{0, m}$ and $\Lambda_{0, m}$. As shown in the Appendix, this can be used to find the general solution of (22) by interpolation of the solution obtained before for kink interaction in the Gardner equation [18]. Correspondingly, the only nontrivial result of interaction in this approximation is a phase shift acquired by each soliton upon collision. The total phase shift $\Delta S_{p}$ for a given $p$ th soliton interacting with all other solitons can be represented by the sum of partial phase shifts $\Delta S_{p q}$ which are due to the collisions of this soliton separately with each of the others. The latter are (see A6):

$$
\begin{aligned}
\Delta S_{p, q}= & \pm\left[\Lambda_{0}^{-1} \ln \left(\frac{2}{\varepsilon_{-} \varepsilon_{q}}\right)^{2}+\left(\Lambda_{m}^{-1}-\Lambda_{0}^{-1}\right) \ln \left(\frac{2}{\varepsilon_{q}}\right)\right. \\
& \left.+\Lambda_{m}^{-1} \ln \left(M_{m} \Lambda_{0}\right)+\Lambda_{0}^{-1} \ln \left(M_{0} \Lambda_{m}\right)\right]
\end{aligned}
$$

where the signs \pm correspond to the cases of $p<q$ and $p>q$, respectively. Thus, an arbitrary $N$-soliton collision can be broken down into pair interactions, so that the effect of multiple collision effects is nonexistent in this approximation.

At the same time, even in the first approximation the nonintegrable case has an interesting specifics. For example, for the solitons in the Gardner equation (Equation (27) at $\Lambda_{0}=\Lambda_{m}=M_{0}=M_{m}=1$ ) we have

$$
\Delta S_{p, q}= \pm \ln \left(\frac{2}{\varepsilon_{p}-\varepsilon_{q}}\right)^{2}
$$

For the MCC solitons considered here, this dependence is supplemented with two additional terms in (27), which can result in a much stronger difference between phase shifts of the solitons at pair interactions. The nature of such a difference is discussed below.

Finally, it should again be underscored that the above results concerning integrability refer only to the first approximation. In general, the MCC Equation (1) are apparently nonintegrable (at least there is no proof of otherwise), which suggests that in the higher approximations, a radiation from the interaction region can be expected. 


\section{Compound solitons and their interactions}

\subsection{A single soliton}

A general approach is illustrated below with two relatively simple examples. We begin from a two-kink solution corresponding to a stationary soliton:

$$
\xi_{s}(x-c t)=\xi_{1}\left(X-S_{1}\right)+\xi_{2}\left(X-S_{2}\right)-\xi_{m}+0(\varepsilon) .
$$

In this case coordinates of kinks, $S_{1,2}(t)$, as follows from (A.1) and (A.2), are:

$S_{1}=-\Lambda_{0}^{-1}[\varepsilon(t-T)+\delta], S_{2}=-\Lambda_{0}^{-1}[\varepsilon(t-T)+\delta]+\Lambda_{m}^{-1}\left[2 \delta+\ln \left(M_{m} \Lambda_{0}\right)\right]$.

Taking into account that $2 \delta=\ln (2 / \varepsilon)$ (see Equation (A.5) in the Appendix) and returning to the dimensional time, one can readily obtain the dependence between the soliton velocity $c$ and the distance between its edge kinks

$$
\sqrt{\Lambda_{0} \Lambda_{m}}\left(c_{m}-c\right) / c_{m}=\Lambda_{0}^{-1} \varepsilon=2 M_{m} e^{-\Lambda_{m}\left(S_{2}-S_{1}\right)} .
$$

Note that this relation can also be obtained from the equations for kink coordinates: $d S_{1,2} / d t=-2 M_{m} \exp \left(-\Lambda_{m}\left(S_{1}-S_{2}\right)\right)$. However, more practical is to use the relation between the soliton velocity $c$ and its amplitude $a$. It follows from the dependence of the distance $S_{2}-S_{1}$ between the kinks on the soliton amplitude. Taking into account that $S_{2}-S_{1} \gg \Lambda_{m}$ and using the asymptotic limits (7) for the kinks, we have

$$
\begin{aligned}
a & =\max \xi_{s}=\left.\xi_{1}\left(X-S_{1}\right)\right|_{X=S_{1}+\left(S_{2}-S_{1}\right) / 2}+\left.\xi_{2}\left(X-S_{2}\right)\right|_{X=S_{2}-\left(S_{2}-S_{1}\right) / 2}-\xi_{m} \\
& =\xi_{m}+2 e_{m} e^{-\Lambda_{m}\left(S_{1}-S_{2}\right) / 2} .
\end{aligned}
$$

Now, eliminating $\left(S_{2}-S_{1}\right)$ from (31) and (32), we obtain

$$
\frac{c(a)}{c_{m}}=1-\frac{M_{m} \sqrt{\Lambda_{0} \Lambda_{m}}\left(a-\xi_{m}\right)^{2}}{2 e_{m}^{2}}
$$

Figure 3 shows this approximate dependence (33) for $c(a)$ together with the exact one, Equation (4). Figure 4 shows soliton profiles according to the approximate (32) and exact (2) solutions. It is seen from these figures that the approximate description agrees very well (with less than a few percent difference) with the exact one in a rather wide range of amplitudes, roughly at $\xi_{m} / 2<a<\xi_{m}$. 


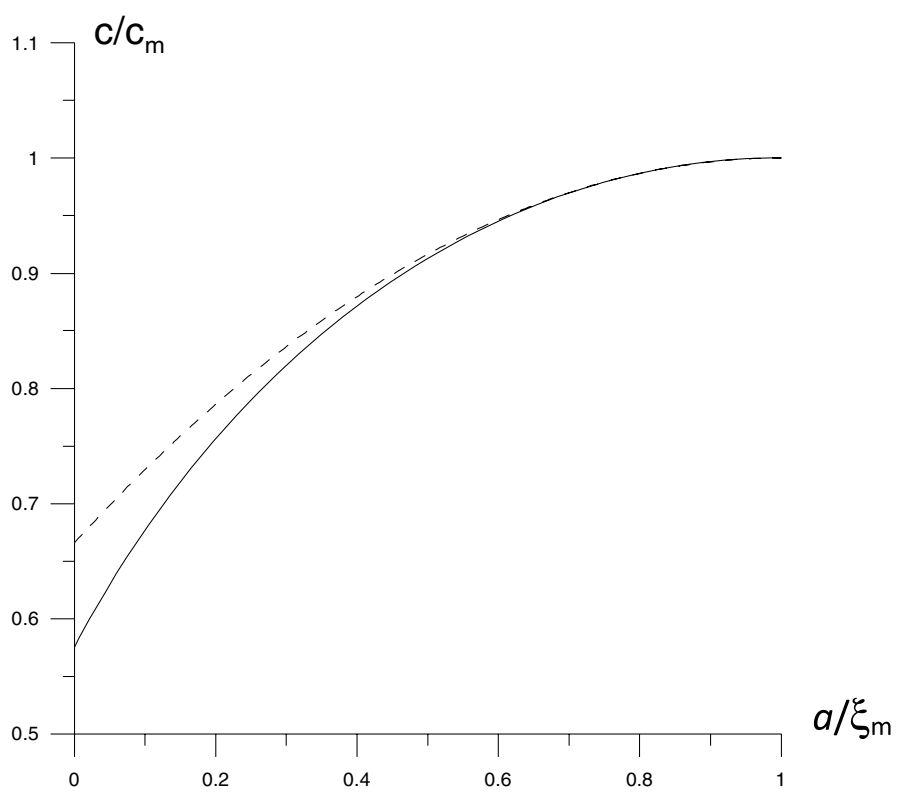

Figure 3. Velocities of the compound (dashed line) and exact (solid line) solitons as functions of amplitude at $h_{1} / h_{2}=0.1, \rho_{1} / \rho_{2}=0.9996, \xi_{m} / h_{1}=-4.495$.

\subsection{Two-soliton interaction}

Consider now the interaction of four kinks corresponding to two interacting solitons:

$$
\xi(x, t)=\sum_{i=1}^{4} \xi_{i}^{(0)}\left(X-S_{i}\right)-2 \xi_{m}+0(\varepsilon)
$$

Here, the expressions for kink coordinates follow from (A.1) and (A.2) at $N=2$ :

$$
\begin{aligned}
& S_{1}=-\Lambda_{0}^{-1} \cdot \ln a_{1}^{(+)}, \\
& S_{2}=-\Lambda_{0}^{-1} \cdot \ln a_{1}^{(+)}+\Lambda_{m}^{-1} \cdot \ln \frac{a_{1}^{(+)}}{a_{1}^{(-)}}+\Lambda_{m}^{-1} \cdot \ln \left(M_{m} \Lambda_{0}\right) . \\
& S_{3}=\Lambda_{0}^{-1} \cdot \ln \frac{a_{1}^{(-)}}{a_{0}^{(+)}}+\Lambda_{m}^{-1} \cdot \ln \frac{a_{1}^{(+)}}{a_{1}^{(-)}}+\Lambda_{m}^{-1} \cdot \ln \left(M_{m} \Lambda_{0}\right)+\Lambda_{0}^{-1} \ln \left(M_{0} \Lambda_{m}\right) \\
& S_{4}=\Lambda_{0}^{-1} \cdot \ln \frac{a_{1}^{(-)}}{a_{0}^{(+)}}+\Lambda_{m}^{-1} \cdot \ln \frac{a_{0}^{(+)}}{a_{0}^{(-)}}+2 \Lambda_{m}^{-1} \cdot \ln \left(M_{m} \Lambda_{0}\right)+\Lambda_{0}^{-1} \cdot \ln \left(M_{0} \Lambda_{m}\right),
\end{aligned}
$$




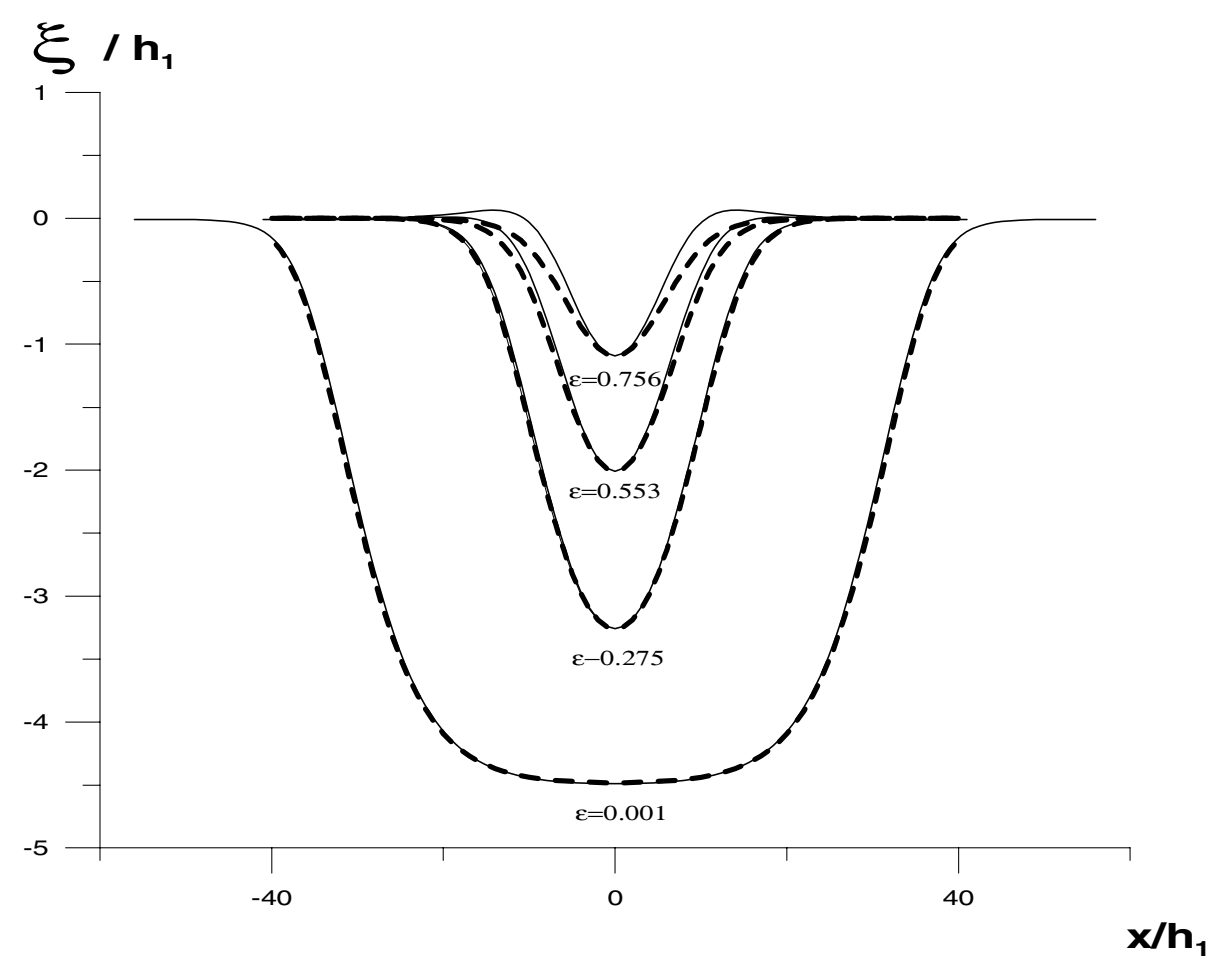

Figure 4. Stationary solutions in Equation (2) at $h_{1} / h_{2}=0.1, \rho_{1} / \rho_{2}=0.9996, \xi_{m} / h_{1}=-4.495$, corresponding to solitary waves with zero asymptotics, at different values of $\varepsilon=\left|\left(a-\xi_{m}\right) / \xi_{m}\right|$. Dashed-lines approximate Equation (41), solid lines exact Equation (2).

where

$$
\begin{aligned}
& a_{1}^{( \pm)}=e^{\varepsilon_{1}\left(t-T_{1}\right) \pm \delta_{1}}+e^{\varepsilon_{2}\left(t-T_{2}\right) \pm \delta_{2}} \\
& a_{0}^{( \pm)}=\exp \left[\varepsilon_{1}\left(t-T_{1}\right)-\delta_{1}+\varepsilon_{2}\left(t-T_{2}\right) \pm \delta_{2}+A_{12}\right] \\
& \delta_{1,2}=\frac{1}{2} \ln \frac{2}{\varepsilon_{1,2}} \\
& A_{12}=\ln \left(\frac{\varepsilon_{1}-\varepsilon_{2}}{2}\right)^{2}, \quad \varepsilon_{1}<\varepsilon_{2} .
\end{aligned}
$$

Soliton collision described by Equation (35) can be considered as a repelling interaction of particles. For example, the expressions for the distance between solitons:

$$
\begin{aligned}
& S_{3}-S_{2}=\Lambda_{0}^{-1} \cdot \ln \left[M_{0} \Lambda_{m} e^{-A_{12}-\delta_{1}-\delta_{2}}\left(\operatorname{ch} \delta+\operatorname{ch} \varepsilon\left(t-T_{0}\right)\right)\right] \\
& \varepsilon=\varepsilon_{2}-\varepsilon_{1}, \quad \delta=\delta_{2}-\delta_{1}, \quad T_{0}=\left(-\varepsilon_{1} T_{1}+\varepsilon_{2} T_{2}\right)\left(\varepsilon_{2}-\varepsilon_{1}\right)^{-1}
\end{aligned}
$$


describe the solitons approaching each other from the infinity up to the minimum distance $\left(S_{3}-S_{2}\right)_{\min }=\Lambda_{0}^{-1} \cdot \ln \left[2 M_{0} \Lambda_{m}\left(\sqrt{\varepsilon_{1}}-\sqrt{\varepsilon_{2}}\right)^{-2}\right]$, which occurs at $t=T_{0}$, after which they diverge. The lengths of solitons vary monotonously at the entire time interval, $-\infty<t<+\infty$ : the length $\left(S_{2}-S_{1}\right)$ of the rear, faster soliton decreases, whereas the length $\left(S_{4}-S_{3}\right)$ of the leading, slower soliton is increasing, namely:

$$
\begin{aligned}
& S_{2}-S_{1}=\Lambda_{m}^{-1} \cdot \ln \left[M_{m} \Lambda_{0} e^{\delta_{1}+\delta_{2}}\left(\operatorname{ch} \delta+\operatorname{sh} \delta \operatorname{th}\left(\varepsilon\left(t-T_{0}\right)-\delta\right) / 2\right)\right] \\
& S_{4}-S_{3}=\Lambda_{m}^{-1} \cdot \ln \left[\left(2 M_{m} \Lambda_{0}\right)^{2} \varepsilon_{1}^{-1} \varepsilon_{2}^{-1}\right]-\left(S_{2}-S_{1}\right) .
\end{aligned}
$$

At $t=T_{0}$, the lengths of these solitons become equal, so that the total field is symmetric in space. Subsequently the solitons diverge and eventually exchange their parameters.

Unlike the differences $S_{2}-S_{1}, S_{3}-S_{2}$, and $S_{4}-S_{3}$, trajectories of individual kinks, $S_{1-4}$, depend significantly on the parameters of solitons, $\varepsilon_{1,2}$. Here two limiting cases with a qualitatively different behavior of kink trajectories can be distinguished.

1. Interaction of solitons with close parameters, $\varepsilon_{2}-\varepsilon_{1} \ll \varepsilon_{1,2}$, for which, as mentioned, the approach developed above results in a dynamic similar to a collision of classical (point) particles. During the entire interaction process, soliton lengths remain close to each other and vary only slightly. The pairs of kinks forming each soliton move almost synchronously (Figure 5), and the resulting phase shift (28) of the solitons is the same as that for point particles with exponential potentials.

2. Interaction of solitons with strongly differing parameters, $\varepsilon_{1} \ll \varepsilon_{2}$. In this case the lengths of the solitons may differ significantly. Moreover, as shown above, the approximate expression (29) for a soliton is valid even for solitons relatively far from limiting, at $\xi_{m} / 2<a<\xi_{m}$. Thus, here one soliton is considered close to the limiting one, whereas the second can be significantly smaller and shorter. For brevity, we call the former a wide (or fast) soliton and the latter, a narrow (or slow) soliton. A typical interaction pattern and trajectories of solitons in this case are shown in Figure 6.

The collision process can be divided into three stages. The first stage begins when the solitons approach each other up to a distance of the order of the length of the narrow, slow soliton. At this stage, the trailing kink of the longer soliton remains far from the other three kinks forming the solitons and is not involved in the interaction. The corresponding solution can be obtained as a degenerated case of (35). Letting $\varepsilon_{1} \rightarrow 0$ in such a way that $\varepsilon_{1} T_{1}=-\delta_{1}$, 


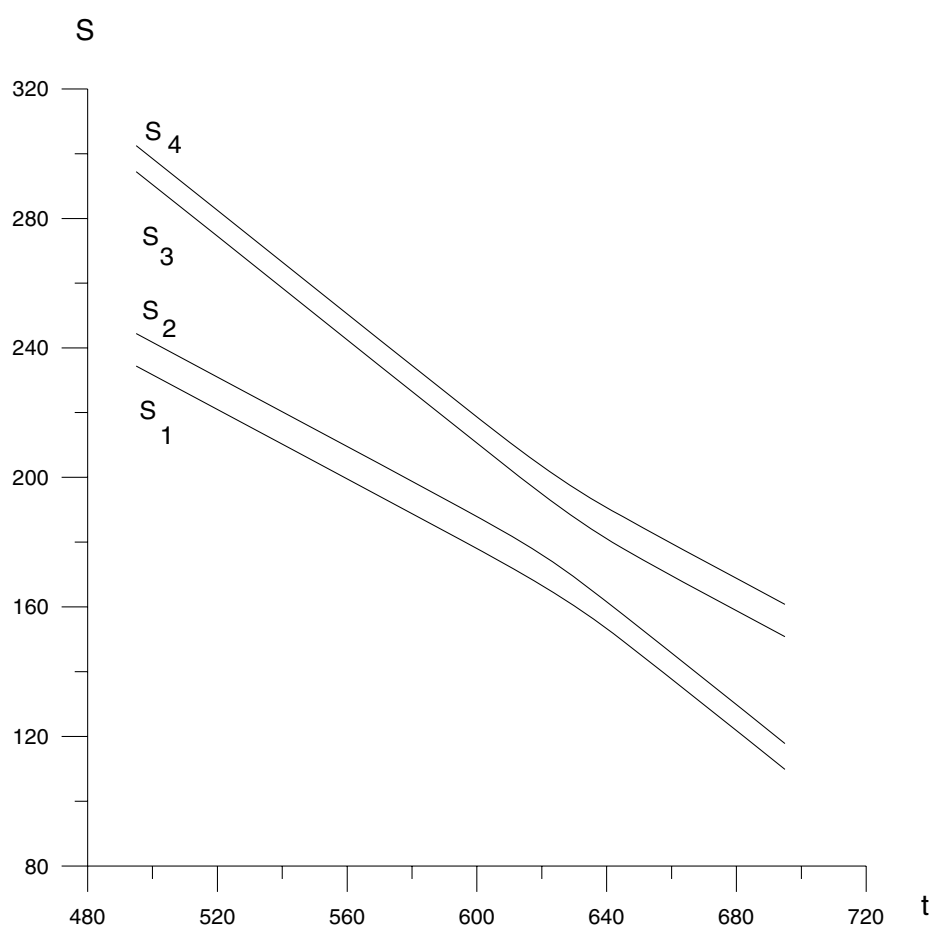

Figure 5. Trajectories of kinks in compound solitons with $a_{1} / h_{1}=-2.4\left(\varepsilon_{1}=0.46\right)$ and $a_{2} / h_{1}=$ $-2\left(\varepsilon_{2}=0.55\right)$, at $h_{1} / h_{2}=0.1, \xi_{m} / h_{1}=-4.49, \rho_{1} / \rho_{2}=0.9996$.

we obtain

$$
\begin{aligned}
S_{2}= & -\Lambda_{m}^{-1} \ln \left(1+e^{\varepsilon_{2}\left(t-T_{2}\right)-\delta_{2}}\right) \\
S_{3}= & -\Lambda_{m}^{-1} \ln \left(1+e^{\varepsilon_{2}\left(t-T_{2}\right)-\delta_{2}}\right)+\Lambda_{0}^{-1} \ln \left(1+e^{-\varepsilon_{2}\left(t-T_{2}\right)+\delta_{2}}\right) \\
& +\Lambda_{0}^{-1} \ln \left(2 M_{0} \Lambda_{m} / \varepsilon_{2}\right) \\
S_{4}= & \Lambda_{0}^{-1} \ln \left(1+e^{-\varepsilon_{2}\left(t-T_{2}\right)+\delta_{2}}\right)+\Lambda_{0}^{-1} \ln \left(2 M_{0} \Lambda_{m} / \varepsilon_{2}\right)+\Lambda_{m}^{-1} \ln \left(2 M_{m} \Lambda_{0} / \varepsilon_{2}\right) .
\end{aligned}
$$

At $t \rightarrow-\infty$, (38) represents the initial disposition of kinks: $S_{2}=0$ is chosen for the front of the faster soliton which is at rest in the reference frame moving with a limiting velocity; for the other kinks at $t \rightarrow-\infty$ we have $S_{3}=\Lambda_{0}^{-1}\left(-\varepsilon_{2}\left(t-T_{2}\right)+\ln \left(2 M_{0} \Lambda_{0} / \varepsilon_{2}\right)+\delta_{2}\right)$ and $S_{4}=$ $-\Lambda_{0}^{-1} \varepsilon_{2}\left(t-T_{2}\right)+\Lambda_{0}^{-1} \ln \left(2 M_{0} \Lambda_{m} / \varepsilon_{2}\right)+\Lambda_{m}^{-1} \ln \left(2 M_{m} \Lambda_{0} / \varepsilon_{2}\right)+\Lambda_{0}^{-1} \delta_{2}$, so that the length of the slower soliton (having a velocity of $-\Lambda_{0}^{-1} \varepsilon_{2}$ ) is $S_{4}-S_{3}=\Lambda_{m}^{-1} \ln \left(2 M_{m} \Lambda_{0} / \varepsilon_{2}\right)$.

As follows from (38), after the collision $(t \rightarrow+\infty)$ the front of the initially slower soliton has accelerated up to the limiting velocity, thus forming the front of the new fast soliton and, in the reference frame used herein, 


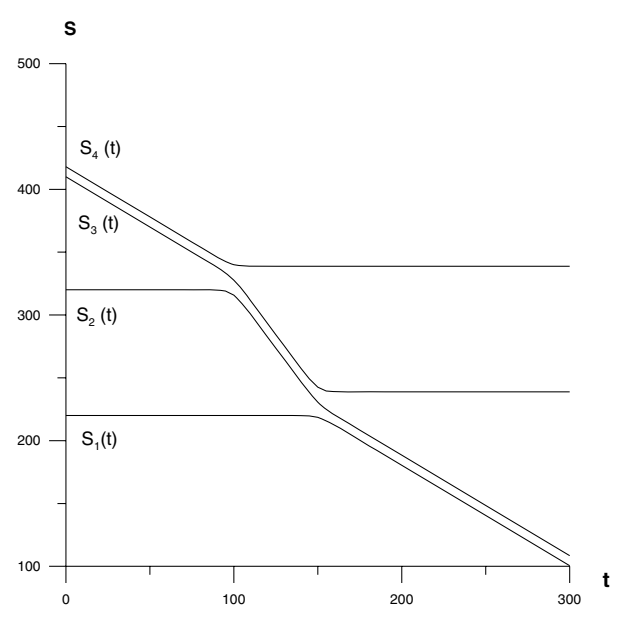

(a)

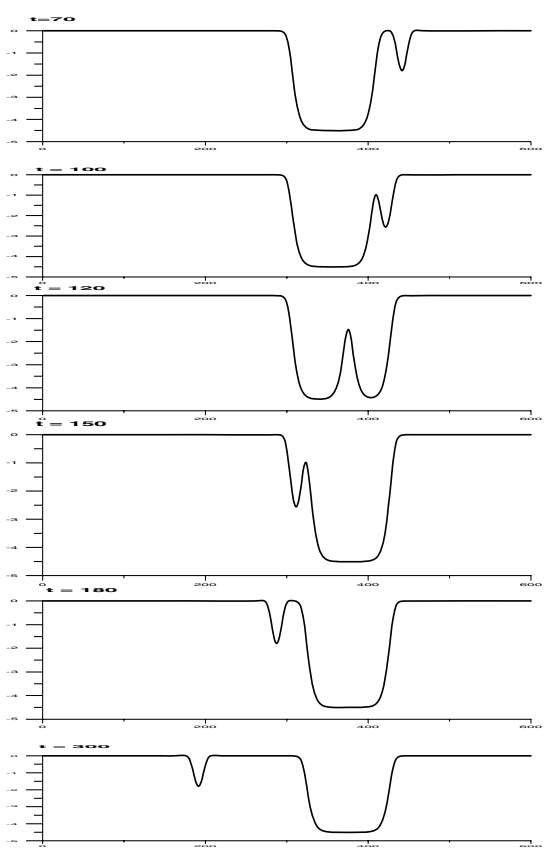

(b)

Figure 6. Interaction of two solitons, one of which is close to a limiting one according to Equation (12). (a) Trajectories $S_{i}(t)$ of four kinks corresponding to interaction of two solitons with amplitudes $a_{1} / h_{1}=-4,48\left(\varepsilon_{1}=2 \cdot 10^{-3}\right)$ and $a_{2} / h_{1}=-2\left(\varepsilon_{2}=0.55\right)$ at $h_{1} / h_{2}=0.1$, $\xi_{m} / h_{1}=-4.49$. (b) Profiles of compound solitons at different time moments.

moving with the velocity $c_{m}$, it stops at the point with the coordinate $S_{4}=\Lambda_{0}^{-1} \ln \left(2 M_{0} \Lambda_{m} / \varepsilon_{2}\right)+\Lambda_{m}^{-1} \ln \left(2 M_{m} \Lambda_{0} / \varepsilon_{2}\right)$.

Other kinks are slowed down and eventually move synchronously; for them $S_{2}=-\Lambda_{m}^{-1}\left(\varepsilon_{2}\left(t-T_{2}\right)-\delta_{2}\right), S_{3}=-\Lambda_{m}^{-1} \cdot\left(\varepsilon_{2}(t-\right.$ $\left.\left.T_{2}\right)-\delta_{2}\right)+\Lambda_{0}^{-1} \ln \left(2 M_{0} \Lambda_{m} / \varepsilon_{2}\right)$.

These kinks form a soliton of the positive polarity opposite to that of the initial solitons; they propagate at a speed of $-\Lambda_{m}^{-1} \varepsilon_{2}$ and have a length $S_{3}-S_{2}=\Lambda_{0}^{-1} \ln \left(2 M_{0} \Lambda_{m} / \varepsilon_{2}\right)$.

At the second stage of interaction, when the positive soliton moves far away from the kinks 1 and $4\left(S_{3}-S_{2} \ll S_{4}-S_{3}, S_{2}-S_{1}\right)$, all kinks move uniformly, and the general pattern can be interpreted as the motion of a narrower positive soliton on the top of the initially wide soliton. The duration of this stage is the longest, so that the duration of the entire interaction process can be estimated as the time of motion of the positive soliton through the initially longer soliton: $(\Delta T)_{\text {int }}=\varepsilon_{2}^{-1} \ln \left(2 M_{m} \Lambda_{0} / \varepsilon_{1}\right)$. 
Finally, at the third stage the positive soliton reaches the trailing edge of the initially long negative soliton 1, and the interaction of kinks 1-3 completes the process of forming the solitons emerging after the collision. The solution describing interaction of these kinks is symmetric with respect to (38) and can be obtained from the latter by changing $t \rightarrow t, S_{4} \rightarrow-S_{1}, S_{3} \rightarrow S_{2}, S_{2} \rightarrow-S_{3}$. From this solution, it follows that the kinks with coordinates $S_{2}$ and $S_{3}$ forming a positive soliton become the front of the newly emerging narrow (slow) soliton and the trailing edge of the long and fast soliton, respectively, whereas the kink at $S_{1}$ is slowing down to become the trailing edge of the slow soliton.

The above description allows one to understand the peculiarities in the dependence of phase shifts of solitons $(\Delta S)_{1,2}$ on the parameters $\varepsilon_{1,2}$ in the case when the lengths of interacting solitons are strongly different. For example, the phase shift $(\Delta S)_{1}$ of the long and fast soliton can already be determined from the first stage of interaction when the final motion of its front is established. As long as only the front of the fast soliton is involved in interaction, it is clear that the result does not depend on its length and, hence, on the parameter $\varepsilon_{1}$ but is determined completely by the parameters of the slower soliton: $(\tilde{\Delta S})_{1}=S_{4}(+\infty)-S_{2}(-\infty)=\Lambda_{0}^{-1} \ln \left(2 M_{0} \Lambda_{m} / \varepsilon_{2}\right)+\Lambda_{m}^{+1} \ln \left(2 M_{m} \Lambda_{0} / \varepsilon_{2}\right)$.

It is interesting that the shift $\Delta S_{1}$ is simply the sum of the lengths of the initial slow soliton and the positive soliton emerging in the process of interaction.

The phase shift of the slow and narrow soliton cumulates at all three stages of the process. Contributions of the first and third stages are of the order of the total length of the slow soliton and the positive soliton. The contribution $\Delta T_{0}$ of the second stage is due to the difference between the time interval $\Delta T_{\text {int }}$ (see above) and the time of movement of this soliton (with the speed $-\varepsilon_{2} \Lambda_{0}^{-1}$ ) through the wide soliton, namely, $\Delta T_{0}=$ $\varepsilon_{2}^{-1} \Lambda_{0} \cdot \Lambda_{m}^{-1} \ln \left(2 M_{m} \Lambda_{0} / \varepsilon_{1}\right)$. The corresponding part of the phase shift can be estimated as $\tilde{\Delta S_{2}}=\varepsilon_{2} \Lambda_{0}^{-1}\left(\Delta T_{0}-\Delta T_{\text {int }}\right)=\left(\Lambda_{m}^{-1}-\Lambda_{0}^{-1}\right) \ln \left(2 M_{m} \Lambda_{0} / \varepsilon_{1}\right)$. It is evident that in the considered range of the parameters $\varepsilon_{1,2}$, the value $\Delta \tilde{S}_{2}$ which is proportional to the length of the fast soliton, makes a determinative contribution to the phase shift $\Delta S_{2}$ of the slow soliton.

The approximate expressions for $\Delta \tilde{S}_{1}$ and $\Delta \tilde{S}_{2}$ are in agreement with the exact expressions for these variables following from (A.6) at $\varepsilon_{1} \ll \varepsilon_{2}$. Divergence of $(\Delta S)_{2}$ at $\varepsilon_{1} \rightarrow 0$ is due to the delay of the positive soliton emerging from the slow soliton at a stage when the distance between the solitons is minimal. It is clear that there is no such singularity if the velocities of these two solitons are the same. This latter case occurs for the solitons in the Gardner equation for which $\Lambda_{m}=\Lambda_{0}=M_{m}=M_{0}=1$. To illustrate this, Figure 7 shows the dependencies of phase shifts $\Delta S_{2}$ for both models, MCC and Gardner, qualitatively differing in their behavior near $\varepsilon_{1} \rightarrow 0$ : due to the long travel on the flat part of the increasingly long large soliton, the phase shift of the smaller one diverges. This effect is absent in the Gardner equation. 


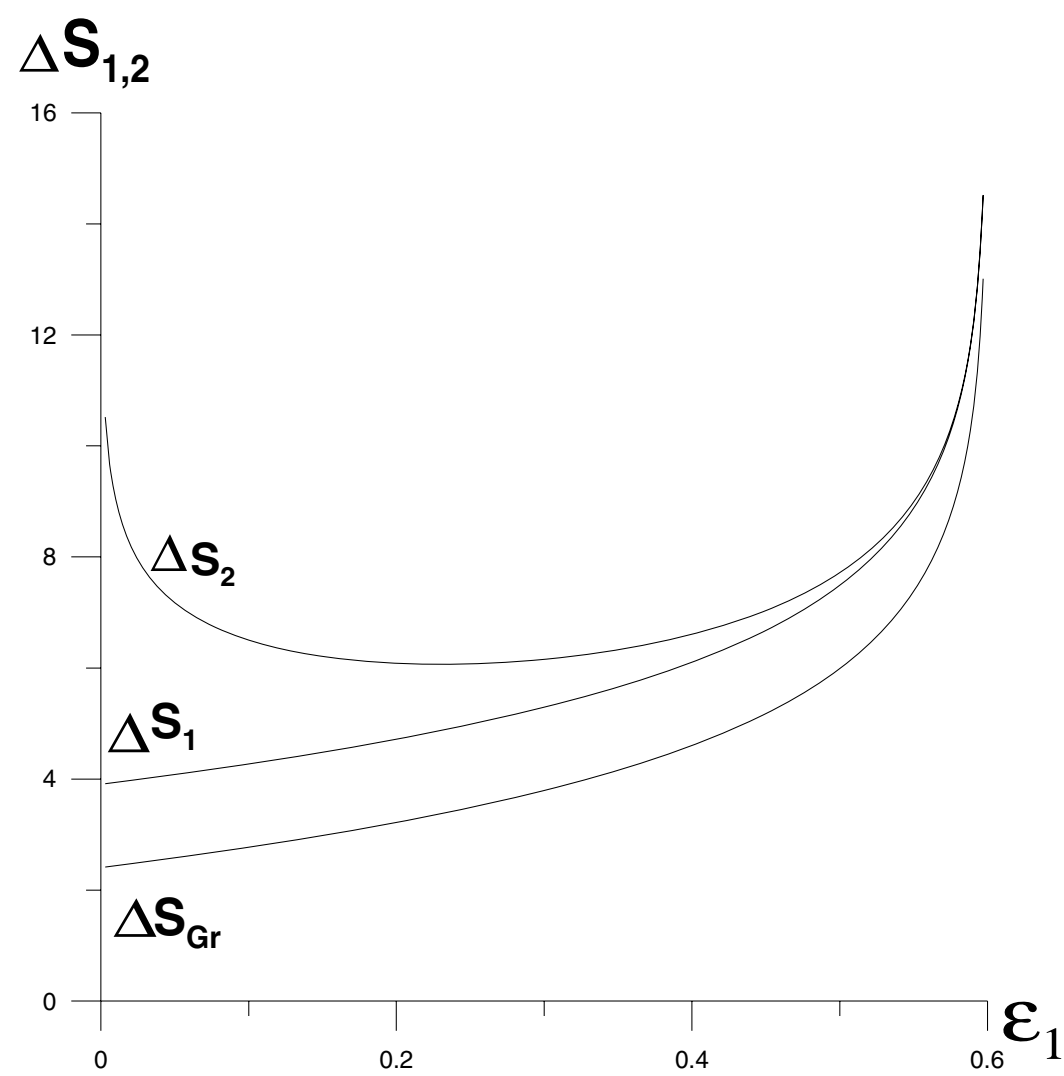

Figure 7. Phase shifts for the fast $\left(\Delta S_{1}\right)$ and slow $\left(\Delta S_{2}\right)$ solitons as functions of $\varepsilon_{1}$ for two interacting solitons with the parameters specified in Figure 6. $\Delta S_{G r}$-phase shift for the same solitons following from the Gardner equation.

\section{Concluding remarks}

We believe that the theory developed here illustrates the efficiency of the perturbation approach for strongly nonlinear equations such as the MCC system; indeed, the integrable dynamical equations obtained in the first approximation do not depend on the exact integrability of the basic equation. Whereas some general features of the soliton interaction described above are similar to those known for integrable models such as the Gardner equation, some interesting specifics are observed even in the main approximation, such as the three-stage process, including the stage when the shorter soliton propagates on the longer, tabletop soliton as on a pedestal; the latter results in an anomalously large phase shift of the smaller soliton.

The description of solitons as compounds of kinks seems to be most adequate for strongly nonlinear waves having the characteristic of approaching a limiting, 
tabletop stage. To illustrate, let us briefly outline the result of application of the earlier theory describing interaction of solitons as whole entities. As follows from the perturbation theory developed in Ref. [16] (which is again independent of the feature of integrability and describes the radiation from the interaction area in the second approximation), in the first approximation the $\mathrm{N}$-soliton interaction is described by a system equivalent to the known Toda lattice:

$$
m d^{2} S_{i} / d t^{2}=\alpha\left[e^{-\Lambda_{0}\left(S_{i}-S_{i-1}\right)}-e^{-\Lambda_{0}\left(S_{i}-S_{i+1}\right)}\right] .
$$

Here $S_{i}$ is the coordinate of a soliton center, $m$ is the equivalent soliton mass that is related to the full momentum $P$ of the soliton as $m=\partial P / \partial c$, and the interaction coefficient $\alpha$ is proportional to the product of exponential asymptotics of neighboring solitons. The integrability of (39) implies that in this approximation the parameters of solitons remain unchanged after the interaction (again, this result is exact only for integrable systems). The only nontrivial results of the interaction are the phase shifts that for each soliton are equal to the sum of the shifts occurring at pair collisions:

$$
\Lambda_{0} \Delta S_{i, j} \operatorname{sign}\left(\dot{S}_{i}-\dot{S}_{j}\right)=\ln \left[2 \Lambda_{0} \partial P / \partial c \mid\left(\dot{S}_{i}-\dot{S}_{j}\right)^{2} / \alpha\right] \mid
$$

(As above, there are no multiple collisions here). The corresponding field structure at each moment is close to the superposition of solitons plus small perturbations:

$$
\begin{aligned}
\xi([x, t)= & \sum_{i=1}\left[\xi^{(0)}+d S_{i} / d t \partial \xi^{(0)} / \partial c+1 / 2\left(d S_{i} / d t\right)^{2} \partial^{2} \xi^{0} / \partial c^{2}\right] \xi^{(0)}=\xi^{(0)}\left(X-S_{i}, c\right) \\
& +\xi^{(2)}+0\left(\varepsilon^{3}\right) .
\end{aligned}
$$

Here, the terms in brackets represent an expansion of the function $\xi^{(0)}\left(X-S_{i}, c+\dot{S}_{i}\right)$ describing the stationary soliton. Note that the term $\xi^{(2)}(X, \tau)$ contains both localized and nonlocalized perturbations, i.e., the radiation.

This representation works well for relatively weak solitons such as those in the $\mathrm{KdV}$ equation to which the MCC system is reduced at small amplitudes. However, for solitons close to limiting it becomes inadequate. Indeed, in such waves very small variations of amplitude and velocity $c$ lead to dramatic variations of their length and, consequently, of the momentum. The derivatives $\partial \Delta / \partial c$ and $\partial p / \partial c$ increase even faster. Thus, the expansions leading to (40) and (41) become ineffective. At the same time, representation of a soliton as a compound of kinks suggested above allows one to extend the perturbation theory to the near-limiting П-solitons.

Note finally, that broadening of a soliton with the growth of its amplitude is a realistic occurrence in oceanography: it is typical of internal waves in a stratified water layer with a sharp pycnocline, for which the MCC and other equations have been formulated. Indeed, the solitary-type waves that are much 
wider than those predicted by weakly nonlinear equations such as the KdV, have been observed more than once in field experiments (see [7] and references therein).

\section{Appendix}

From (26) it follows that the solution of (22) expressed in terms of $R_{\mathrm{i}}(t)$, has the same form at any values of the parameters $M_{0, m}$ and $\Lambda_{0, m}$. This can be used to find the general solution of (22), explicitly depending on the parameters $M_{0, m}$ and $\Lambda_{0, m}$ by using their solutions known for the particular case $M_{m}=M_{0}=1, \Lambda_{m}=\Lambda_{0}=1$ which corresponds to kink interaction in the Gardner equation $[18,19]$ :

$$
\begin{aligned}
\underset{\substack{i=2 p-1 \\
(p=1,2,3, . .)}}{S_{0}} & \frac{1}{\Lambda_{0}} \ln \left[\frac{a_{N-p+1}^{(-)}}{a_{N-p}^{(+)}}\right] \cdot+\frac{1}{\Lambda_{m}} \ln \left[\frac{a_{N-p+1}^{(+)}}{a_{N-p+1}^{(-)}}\right]+(p-1) \\
& \times\left[\Lambda_{m}^{-1} \ln \left(\Lambda_{0} M_{m}\right)+\Lambda_{0}^{-1} \ln \left(\Lambda_{m} M_{0}\right)\right] \\
\underset{\substack{i=2 p \\
(p=1,2,3, . .)}}{S_{0}} & \frac{1}{\Lambda_{0}} \ln \left[\frac{a_{N-p+1}^{(-)}}{a_{N-p}^{(+)}}\right]+\frac{1}{\Lambda_{m}} \ln \left[\frac{a_{N-p}^{(+)}}{a_{N-p}^{(-)}}\right]+p \Lambda_{m}^{-1} \ln \left(\Lambda_{0} M_{m}\right) \\
& +(p-1) \Lambda_{0}^{-1} \ln \left(\Lambda_{m} M_{0}\right) .
\end{aligned}
$$

Here the functions $a_{\sigma}^{( \pm)}(t)$ are defined as follows:

$$
\begin{aligned}
& s_{i=2 p-1}=\ln \left[\frac{a_{N-p+1}^{(+)}}{a_{N-p}^{(+)}} .\right] ; \quad s_{i=2 p}=\ln \left[\frac{a_{N-p+1}^{(-)}}{a_{N-p}^{(-)}}\right] \\
& a_{N}^{( \pm)}=1, \quad a_{N-1}^{( \pm)}=\sum_{j=1}^{N} \exp \left[\varepsilon_{j}\left(t-T_{j}\right) \pm \delta_{j}\right] \\
& a_{N-2}^{( \pm)}(t)=\sum_{1<j_{1}<j_{2}}^{N} \exp \left[\varepsilon_{j_{1}}\left(t-T_{j 1}\right)+\varepsilon_{j_{2}}\left(t-T_{j_{2}}\right) \pm \delta_{j_{1}} \pm \delta_{j_{2}} \pm A_{j_{1, j 2},},\right. \\
& a_{N-p}^{( \pm)}(t)=\sum_{1<j_{1}<j_{2} \ldots j_{p}}^{N} \exp \left[\sum_{k=1}^{p}\left[\varepsilon_{j_{k}}\left(t-T_{j_{k}}\right) \pm \delta_{j_{k}}\right]+\sum_{1<k<l}^{p} A_{j l, j k,}\right], \\
& a_{0}^{( \pm)}=\exp \left[\sum_{j=1}^{N} \exp \left[\varepsilon_{j}\left(t-T_{j}\right) \pm \delta_{j}\right]+\sum_{1<j<j_{l}}^{N} A_{j, j 1}\right],
\end{aligned}
$$


where $\delta_{j}=\left(\ln \left(2 / \varepsilon_{j}\right)\right) / 2, \exp A_{j_{k} j_{l}}=\left(\varepsilon_{j_{k}}-\varepsilon_{j_{l}}\right)^{2} / 4$, and $\varepsilon_{j}$ and $T_{j} \quad(j=1$, $2 \ldots N)$ are a set of $2 N$ independent parameters characterizing relative velocities and positions of solitons.

Note first that the sum of exponents in the expressions for $a_{N-p}^{( \pm)}(t)$ at large times can be significantly simplified, because at $t \rightarrow-\infty$ the exponent of a minimal power becomes dominant in the sum, whereas at $t \rightarrow+\infty$ another exponent, having a maximal power, would dominate. These exponents depend on a different set of $p$ parameters $\varepsilon_{j}$. Without lack of generality one can range the parameters $\varepsilon_{j}$ such as $\varepsilon_{1}<\varepsilon_{2}<\cdots<\varepsilon_{N-1}<\varepsilon_{N}$, so that the minimum and maximum of the exponent powers for each $p$ are, respectively, $\sum_{j=1}^{p} \varepsilon_{j}$ and $\sum_{j=N-p+1}^{N} \varepsilon_{j}$, and the functions $a_{N-p}^{( \pm)}(t)$ have the following asymptotics:

$$
\begin{aligned}
& a_{N-p}^{( \pm)}=\exp \left[\sum_{j=1}^{p}\left[\varepsilon_{j}\left(t-T_{j}\right) \pm \delta_{j}\right]+\sum_{1<k<l}^{p} A_{k, l}\right], \quad t \rightarrow-\infty \\
& a_{N-p}^{( \pm)}=\exp \left[\sum_{j=N-p+1}^{N}\left[\varepsilon_{j}\left(t-T_{j}\right) \pm \delta_{j}\right]+\sum_{N-p+1<J<j_{1}}^{N} A_{j, j_{1}}\right], \quad t \rightarrow+\infty .
\end{aligned}
$$

Substitution of these expressions into (A.1) yields the following formulae for the functions $R_{i=2 p-1}$ at large times:

$$
\underset{\substack{i=2 p-1 \\
p=1,2, . . N}}{R^{i}}=\left\{\begin{array}{ll}
2 \delta p, & t \rightarrow-\infty \\
2 \delta_{N-p+1}, & t \rightarrow+\infty
\end{array}\right\}
$$

which is actually the proof of the result formulated above: an initial (at $t \rightarrow-\infty)$ set of solitons with the distances $S_{2 p}-S_{2 p-1}=\Lambda_{m}^{-1}\left(2 \delta_{p}+\ln \left(\Lambda_{0} M_{m}\right)\right)$ between their kinks (edges) is reproduced after interaction so that $S_{2 p}-S_{2 p-1}=\Lambda_{m}^{-1}\left(2 \delta_{N-p+1}+\ln \left(\Lambda_{0} M_{m}\right)\right)$ at $t \rightarrow+\infty$, but with the inverse order of solitary waves. The only nontrivial factor is phase shifts acquired by solitons upon collision. To find these phase shifts, it is sufficient to consider the asymptotics of coordinates of kinks of the same polarity which, in the limits of $t \rightarrow \pm \infty$, belong to the solitons with the same velocities (the same parameter $\varepsilon_{p}$ ). By comparing these asymptotics, we obtain an expression for the shifts of the kink centers (i. e., phase shifts):

$$
\begin{aligned}
\Delta S_{p}= & S_{2(N-p)+1}(t \rightarrow+\infty)-S_{2 p-1}(t \rightarrow-\infty) \\
= & \Lambda_{0}^{-1}\left(\sum_{q=1}^{p-1} A_{p q}-\sum_{q=p+1}^{N} A_{p q}\right)-2\left(\Lambda_{m}^{-1}-\Lambda_{0}^{-1}\right)\left(\sum_{q=1}^{p-1} \delta_{q}-\sum_{q=p+1}^{N} \delta_{q}\right) \\
& +(N-2 p+1)\left[\left(\Lambda_{0}\right)^{-1} \ln \left(\Lambda_{m} M_{0}\right)++\Lambda_{m}^{-1} \ln \left(\Lambda_{0} M_{m}\right)\right] .
\end{aligned}
$$


As follows from (A.5), the total phase shift $\Delta S_{p}$ for a given $p$ th soliton is equal to the sum of partial phase shifts $\Delta S_{p q}$ which are due to the collisions of this soliton separately with each of the others, namely,

$$
\begin{aligned}
\Delta S_{p, q}= & \pm\left[\Lambda_{0}^{-1} \ln \left(\frac{2}{\varepsilon_{-} \varepsilon_{q}}\right)^{2}+\left(\Lambda_{m}^{-1}-\Lambda_{0}^{-1}\right) \ln \left(\frac{2}{\varepsilon_{q}}\right)\right. \\
& \left.+\Lambda_{m}^{-1} \ln \left(M_{m} \Lambda_{0}\right)+\Lambda_{0}^{-1} \ln \left(M_{0} \Lambda_{m}\right)\right]
\end{aligned}
$$

where + and - correspond to the cases of $p<q$ and $p>q$, respectively.

\section{References}

1. K. V. Konyaev, K. D. Sabinin, and A. D. Serebryany, Two ways for generation of intensive internal waves near an underwater ridge, Proc.(Doklady) Russian Acad. Sci. 338:537-540 (1994).

2. T. P. Stanton and L. A. Ostrovsky, Observations of highly nonlinear internal solitary waves over the continental shelf, Geophys. Res. Lett. 25:2695-2698 (1998).

3. M. TRevorrow, Observations of internal solitary waves near the Oregon Coast with an inverted echo sounder, J. Geophys. Res. 103:7671-7680 (1998).

4. R. Kropfli, L. Ostrovsky, A. Smirnov, E. Skirta, A. Keane, and V. Irisov, Relationships between strong internal waves in the coastal zone and their radar signatures, J. Geophys. Res. 104:3133-3148 (1999).

5. P. Holloway, E. Pelinovsky, and T. Talipova, Internal tide transformation and oceanic internal solitary waves, in Environmental Stratified Flows (R. Grimshaw, Ed.), Ch. 2, pp. 29-60, Kluwer, Norwell, MA, 2002.

6. K. D. SAbinin and A. N. Serebryany, Intense short-period internal waves in the ocean. J. Marine Res. 63:227-261 (2005).

7. J. R. Apel, L. A. Ostrovsky, Y. A. Stepanyants, and J. F. Lynch, Internal solitons in the ocean and their effect on underwater sound, J. Acoust. Soc. Am. 121:695-722 (2007).

8. CH-Y. LeE and R. C. BeARDSLEY, The generation of long nonlinear internal waves in a weakly stratified shear flows, J. Geophys. Res. 79:453-457 (1974).

9. R. Grimshaw, Internal solitary waves, in Environmental Stratified Flows (R. Grimshaw, Ed.), Ch. 1, pp. 1-28, Kluwer, Norwell, MA, 2002.

10. M. MiYatA, Long internal waves of large amplitude, in Nonlinear water waves (K. Horikawa and H. Maruo, Eds.), Springer-Verlag, 1988.

11. W. Chо and R. CAmassa, Fully nonlinear internal waves in a two-fluid system, J. Fluid. Mech. 396:1-36 (1999).

12. L. Ostrovsky and J. Grue, Evolution equations for strongly nonlinear internal waves, Phys. Fluids 15:2934-2948 (2003).

13. A. Slyunyaev and E. Pelinovsky, Dynamics of large-amplitude solitons, J. Exp. Theor. Phys. 89:173-181 (1999).

14. T. Jo and W. CHOI, Dynamics of strongly nonlinear internal solitary waves in shallow water, Stud. Appl. Math. 109:205-228 (2002).

15. K. A. GorshKov and L. A. OstrovsKY, Interactions of solitons in nonintegrable systems: direct perturbation method and applications, Physica D. 3:428-438 (1981). 
16. L. Ostrovsky and K. GorshKov, Perturbation theories for nonlinear waves, in Nonlinear Science at the Dawn of the 21st Century (P. L. Christiansen, M. P. Sorensen, and A. C. Scott, Eds.), pp. 47-65, Springer-Verlag, Berlin, 2000.

17. K. A. Gorshkov and I. A. Soustova, Interaction of solitons as compound structures in Gardner model, Radiophys. Quant. Electronics 14:465-476 (2001).

18. K. A. Gorshrov, I. A. Soustova, L. A. Ostrovsky, and V. G. Irisov, Perturbation theory for kinks and application for multisoliton interactions in hydrodynamics, Phys. Rev. E. 69:1-9 (2004).

19. S. P. Novikov, S. V. Manakov, L. P. Pitaevskit, and V. E. Zakharov, Theory of Solitons: The Inverse Scattering Method, Springer-Verlag, Berlin, 1984.

Russian ACAdEmy of ScIEnce, Nizhny Novgorod, Russia

Russian Academy of Science, Nizhny Novgorod, Russia and NOAA Earth System

Research Laboratory, Boulder, Colorado

Russian ACADEMy of ScIEnCE, Nizhny Novgorod, Russia

(Received August 5, 2010) 